\title{
Integrative Functional Genomic Analysis of Molecular Signatures and Mechanistic Pathways in the Cell Cycle Underlying Alzheimer's Disease
}

\author{
Zhike Zhou ${ }^{D},{ }^{1}$ Jun Bai, ${ }^{2}$ Shanshan Zhong, ${ }^{3}$ Rongwei Zhang, ${ }^{1}$ Kexin Kang, \\ Xiaoqian Zhang, ${ }^{3}$ Ying $\mathrm{Xu},{ }^{2,4}$ Chuansheng Zhao $₫{ }^{3}$ and Mei Zhao $\oplus^{5}$ \\ ${ }^{1}$ Department of Geriatrics, The First Affiliated Hospital, China Medical University, Shenyang, 110001 Liaoning, China \\ ${ }^{2}$ Cancer Systems Biology Center, The China-Japan Union Hospital, Jilin University, Changchun, 130033 Jilin, China \\ ${ }^{3}$ Department of Neurology, The First Affiliated Hospital, China Medical University, Shenyang, 110001 Liaoning, China \\ ${ }^{4}$ Computational Systems Biology Lab, Department of Biochemistry and Molecular Biology and Institute of Bioinformatics, \\ University of Georgia, USA \\ ${ }^{5}$ Department of Cardiology, The Shengjing Affiliated Hospital, China Medical University, Shenyang, 110004 Liaoning, China
}

Correspondence should be addressed to Chuansheng Zhao; cszhao@cmu.edu.cn and Mei Zhao; zhaom1@sj-hospital.org

Received 27 February 2021; Accepted 25 June 2021; Published 12 July 2021

Academic Editor: Giulia Sita

Copyright (C) 2021 Zhike Zhou et al. This is an open access article distributed under the Creative Commons Attribution License, which permits unrestricted use, distribution, and reproduction in any medium, provided the original work is properly cited.

\begin{abstract}
Objective. Alzheimer's disease (AD) is associated with cell cycle reentry of mature neurons that subsequently undergo degeneration. This study is aimed to identify key regulators of the cell cycle and their underlying pathways for developing optimal treatment of AD. Methods. RNA sequencing data were profiled to screen for differentially expressed genes in the cell cycle. Correlation of created modules with $\mathrm{AD}$ phenotype was computed by weight gene correlation network analysis (WGCNA). Signature genes for trophic factor receptors were determined using Pearson correlation coefficient (PCC) analysis. Results. Among the 13,679 background genes, 775 cell cycle genes and 77 trophic factor receptors were differentially expressed in $\mathrm{AD}$ versus nondementia controls. Four coexpression modules were constructed by WGCNA, among which the turquoise module had the strongest correlation with AD. According to PCC analysis, 10 signature trophic receptors most strongly interacting with cell cycle genes were filtered and subsequently displayed in the global regulatory network. Further cross-talking pathways of signature receptors, such as glutamatergic synapse, long-term potentiation, PI3K-Akt, and MAPK signaling pathways, were identified. Conclusions. Our findings highlighted the mechanistic pathways of signature trophic receptors in cell cycle perturbation underlying AD pathogenesis, thereby providing new molecular targets for therapeutic intervention in AD.
\end{abstract}

\section{Introduction}

Alzheimer's disease (AD) accounts for $60-70 \%$ of all dementia in the elderly and thus imposes a heavy burden on society $[1,2]$. This clinical entity is a slowly progressing brain disorder manifested by cognitive decline, behavioral abnormality, and psychiatric alteration [3]. Pathologically, the core hallmarks of $\mathrm{AD}$ are neurofibrillary tangles composed of hyperphosphorylated tau and senile plaques consisting of betaamyloid (A $\beta$ ) peptides [4]; besides, synaptic and neuronal deficits are also characteristic of the disease $[5,6]$. Neuron demise in $\mathrm{AD}$ has been proposed to be attributable, at least in part, to abnormal activation of cell cycle proteins and DNA tetraploidy followed by neuronal reentry into the cell cycle [2]. Indeed, the cell cycle is a tightly regulated process that, when reinitiated or undergoing failure of cell cycle arrest, may result in a pattern of programmed cell death termed as apoptosis $[2,7,8]$. This unscheduled event in turn facilitates $\mathrm{A} \beta$ toxicity and tau hyperphosphorylation, thus to the formation of $\mathrm{AD}$ neuropathology $[9,10]$. 
TABLE 1: The number of samples for both $\mathrm{AD}$ and non-dementia controls in included datasets.

\begin{tabular}{lcccc}
\hline Tissue & GEO & Platform & AD & Controls \\
\hline & GSE132903 & GLP10558 & 97 & 98 \\
& GSE118553 & GPL10558 & 45 & 24 \\
Temporal lobe & GSE5281 & GPL570 & 16 & 11 \\
& GSE37264 & GPL5188 & 8 & 8 \\
& GSE36980 & GPL6244 & 10 & 19 \\
\hline
\end{tabular}

AD: Alzheimer's disease; GEO: Gene Expression Omnibus.

Progress in the cell cycle depends on the coordination and interaction of trophic factors with two categories of regulatory proteins, including cyclins and cyclin-dependent kinases (CDKs) $[11,12]$. For instance, nerve growth factor (NGF) inhibits the induction of cyclins and their associations with specific CDKs, hence interrupting cell cycle reentry through the G1-phase [13]. Insulin-like growth factor 1 (IGF-1) prevents the expression of cell cycle proteins (e.g., cyclin A, D1, and CDK2) known to stimulate G1 quiescent cells to enter the S-phase [14]. Notably, both NGF and IGF1 have been found to be downregulated in $\mathrm{AD}$, with the extent of downregulation proportional to $\mathrm{A} \beta$ burden and poor cognition $[15,16]$. Additional evidence also supports the regulatory role of trophic factors in the cell cycle, such as epidermal growth factor [17], endothelial growth factor [18], and fibroblast growth factor [19]. Intriguingly, the expression of corresponding receptors for these factors presents periodic variations throughout the cell cycle, giving rise to intermittent effects of the ligands on neurons at different phases of the cell cycle [20].

Based on such observations, we preliminarily infer that neuronal death following cell-cycling initiation is implicated in $\mathrm{AD}$ pathogenesis, at least partially due to insufficient support from trophic factors (either downregulation or intermittent effect). This is of profound clinical significance, as understanding AD neurodegeneration mediated by early trophic receptor processing and signaling events in the cell cycle offers promise for those who seek therapeutic interventions, which may contribute to slowing or even blocking the occurrence and progression of AD. Accordingly, we collected cell cycle-encoding genes as well as trophic factor receptors in incident $\mathrm{AD}$ dementia by reviewing existing literature. Subsequently, an integrative genomic analysis was performed on basis of gene expression profiles and functional annotations, aiming to (1) provide a computational validation for the involvement of cell cycle in AD onset, (2) identify key regulators as attractive therapeutic targets in a cell cycle subnetwork, and (3) elucidate the underappreciated therapeutic targets in the pathogenesis of $\mathrm{AD}$.

\section{Materials and Methods}

2.1. Data Resources. All RNA sequencing (RNA-seq) and microarray data of temporal cortex tissues from $\mathrm{AD}$ patients and nondementia controls were downloaded through Gene Expression Omnibus (GEO, https://www.ncbi.nlm.nih.gov/ geo/) database [21]. Table 1 exhibited the information of selected datasets and the number of samples for analysis. Sex-matching was observed between $176 \mathrm{AD}$ cases (male/female: 89/87) and 160 nondementia controls (male/female: $85 / 75 ; p=0.64$ ). The mean age was $83.60 \pm 8.25$ years (range: 40-105 years) for AD and $81.38 \pm 10.78$ years (range: $43-102$ ) for nondementia. Clinical phenotypic data of samples are detailed in Supplementary Table S1. Subjects were clinically and/or pathologically diagnosed with $\mathrm{AD}$ had to be based on certain standardized criteria, e.g., the National Institute of Neurological and Communicative Disorders and StrokeAlzheimer's Disease and Related Disorders Association (NINCDS-ADRDA) [22]; the International Classification of Diseases- (ICD-) 10 criteria, and the Diagnostic and Statistical Manual of Mental Disorders- (DSM-) III, -IV, or -V criteria [23-26]; the Consortium to Establish a Registry for Alzheimer's Disease (CERAD) guidelines [27]; and the Braak stage [4]. The list of cell cycle genes was obtained from a published study (see Supplementary Table S2) [28]. A total of 169 receptors for cell cycle-related trophic factors was collected based on our literature review (see Supplementary Table S3).

2.2. Gene Set Enrichment Analysis (GSEA). Gene expression profiles from five datasets (GSE132903, GSE118553, GSE5281, GSE37264, and GSE36980) were merged into a new dataset. The limma package of $\mathrm{R}$ software was used to eliminate batch effects during the merging process. As shown in Table 2, biological processes (BP) of gene ontology (GO) terms significantly enriched in $\mathrm{AD}$ phenotype were filtered by GSEA $[29,30]$. The number of permutations was set to 1000 , and $p<0.05$ was considered statistically significant. The visualization of GSEA was accomplished using ClusterProfler, enrichplot, ggplot2, and GSEABase packages.

2.3. Identification of Differentially Expressed Genes (DEGs). Differential expression analysis of genes was conducted by comparing RNA-seq data between $\mathrm{AD}$ and control tissues adopting $l m$ Fit and eBayes functions. Analyses of twodimensional hierarchical clustering and volcano plot for DEGs were performed using limma. A false discovery rate(FDR-) adjusted $p<0.05$ and fold change (FC) $\geq 1.2$ were defined as differentially expressed [31-33].

2.4. Coexpression Network Analysis and Signature Trophic Receptors. The cell cycle genes (Supplementary Table S2) and trophic receptors (Supplementary Table S3) obtained from our literature review were merged to match the DEGs. The overlapping genes (defined as cell cycle-related genes) and their coexpression modules were identified by weighted correlation network analysis (WGCNA) [34], with the differential expression of genes given in Supplementary Table S4. During clustering analyses, the genes that were heavily involved in noncell cycle processes were grouped into the grey module, as their expression levels might not necessarily represent the actual level of cell cycle activity in AD. The correlation of cell cycle genes with trophic receptors was computed by Pearson correlation coefficient (PCC). The association of cell cyclerelated genes with their phenotypes (i.e., $\mathrm{AD}$, age, and 
TABLE 2: Biological processes of gene set enrichment analysis.

\begin{tabular}{lccc}
\hline ID & Description & Enrichment score & $p$ value \\
\hline GO:0007267 & Cell-cell signaling & -0.339309387 & 0.002159827 \\
GO:0051649 & Establishment of localization in cell & -0.333291297 & 0.002183406 \\
GO:0042127 & Regulation of cell proliferation & 0.329444498 & 0.001858736 \\
GO:1902679 & Negative regulation of RNA biosynthetic process & 0.328198078 & 0.001992032 \\
GO:1903507 & Negative regulation of nucleic acid-templated transcription & 0.328198078 & 0.001992032 \\
GO:0003008 & System process & -0.32530293 & 0.001996008 \\
GO:0043067 & Regulation of programmed cell death & 0.321860897 & 0.001941748 \\
GO:0055085 & Transmembrane transport & -0.319180234 & 0.00204918 \\
GO:0051253 & Negative regulation of RNA metabolic process & 0.31829589 & 0.001945525 \\
GO:0042981 & Regulation of apoptotic process & 0.318025216 & 0.001956947 \\
\hline
\end{tabular}

TABLE 3: PCC and GS of signature trophic receptors.

\begin{tabular}{lcccr}
\hline Receptor & moduleColor & PCC.cellcycle & GS.AD & p.GS.AD \\
\hline GABRG2 & Turquoise & 0.419343809 & -0.4121841 & $4.61621 \mathrm{E}-14$ \\
PGRMC1 & Turquoise & 0.405662742 & -0.3663381 & $3.24182 \mathrm{E}-11$ \\
EPHA4 & Turquoise & 0.404438747 & -0.3652521 & $3.74087 \mathrm{E}-11$ \\
MAGED1 & Turquoise & 0.404022816 & -0.3860747 & $2.18463 \mathrm{E}-12$ \\
GRIA2 & Turquoise & 0.394280106 & -0.3667586 & $3.06654 \mathrm{E}-11$ \\
CHRM1 & Turquoise & 0.381313001 & -0.3271977 & $4.06247 \mathrm{E}-09$ \\
TNFRSF1B & Turquoise & 0.353972271 & 0.3201443 & $9.04973 \mathrm{E}-09$ \\
GRIN2A & Turquoise & 0.352962267 & -0.4108274 & $5.68839 \mathrm{E}-14$ \\
TNFRSF1A & Turquoise & 0.341193899 & 0.3440933 & $5.47841 \mathrm{E}-10$ \\
RXRA & Turquoise & 0.339039633 & 0.3940831 & $6.93657 \mathrm{E}-13$ \\
\hline
\end{tabular}

AD: Alzheimer's disease; GS: gene significance; PCC: Pearson correlation coefficient.

gender) was measured by gene significance (GS). Under the premise of p.GS $<0.05$ in $\mathrm{AD}$ phenotype, the top 10 receptors were identified as the signature trophic receptors, the expression of which had the strongest correlation with cell cycle genes (Table 3). Detailed data of PCC and GS for each receptor are shown in Supplementary Table S5. Functional enrichment analyses of Kyoto Encyclopedia of Genes and Genomes (KEGG) pathways were carried out using clusterProfiler.

2.5. Global Regulatory Network and Cross-Talking Pathways of Signature Trophic Receptors. Based on the STRING database (Search Tool for the Retrieval of Interacting Genes, https://www.string-db.org/) [35], the module with the strongest correlation with $\mathrm{AD}$ was selected to construct the global regulatory network. Thereafter, cytoscape software was utilized to visualize the global regulatory network and cross-talking pathways of signature trophic receptors [36].

2.6. Analysis of Area under the Curve (AUC). The $p R O C$ function was adopted to estimate the diagnostic performance of signature trophic receptors in differentiating $\mathrm{AD}$ from controls. Generally, a complete prediction was indicated by an AUC value of $100 \%$, whereas a random selection was represented by $50 \%$. All $p$ values were bilateral with $p<0.05$ considered of statistical significance.

\section{Results}

3.1. Differentially Expressed Genes and GESA of Biological Processes. After removing unannotated and duplicate genes, 6,847 out of 13,679 background genes were differentially expressed between $\mathrm{AD}$ and nondementia controls (see Methods) (Figure 1(a)). There were 852 cell cycle-related genes (including 775 cell cycle genes and 77 trophic receptors) that overlapped with these DEGs (Supplementary Table S4). Heatmap of DEGs with the top 25 up- and downregulated expression in $\mathrm{AD}$ and nondementia controls was exhibited in Figure 1(b). In $\mathrm{AD}$, the major enrichment of BP (Table 2) was involved in the regulation of cell proliferation, programmed cell death, and apoptotic processes, as well as negative regulation of nucleic acid-templated transcription, RNA biosynthetic, and metabolic processes.

3.2. Coexpression Modules and Functional Enrichment Analysis. Three hundred and eight samples passed the cut-off line with a height of 25 , which were hierarchically clustered by the average linkage. Four created modules were established by WGGNA, among which the grey module was composed of noncoexpressed genes, indicating them to be involved in noncell cycle processes. Heatmap of module-trait relationships (Figure 2(a)) presented the most significant negative correlation of turquoise module (correlation coefficient $=-0.45, p=1 e-16)$, as well as a 


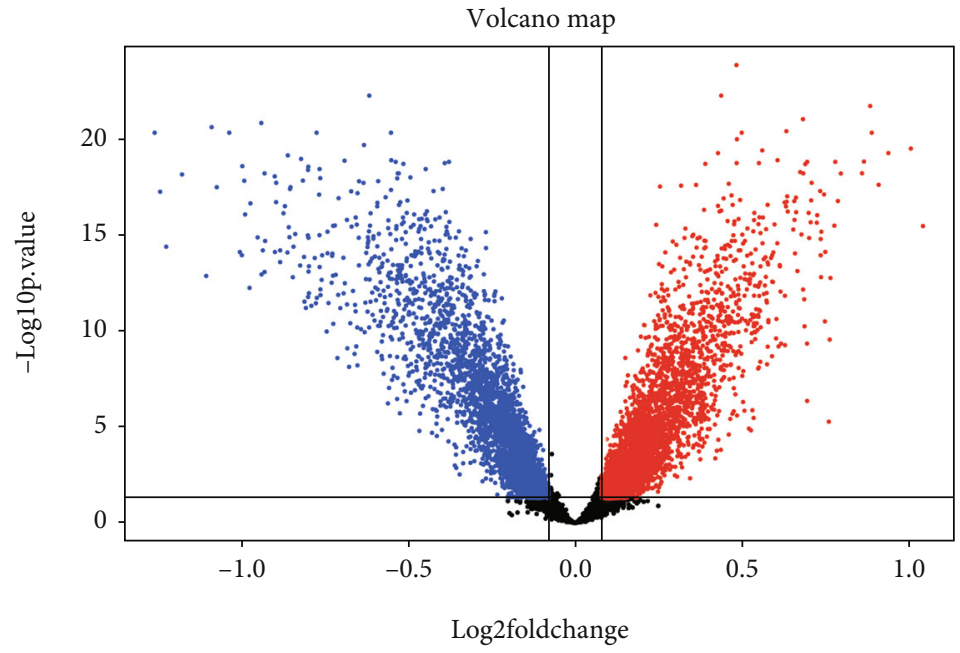

(a)

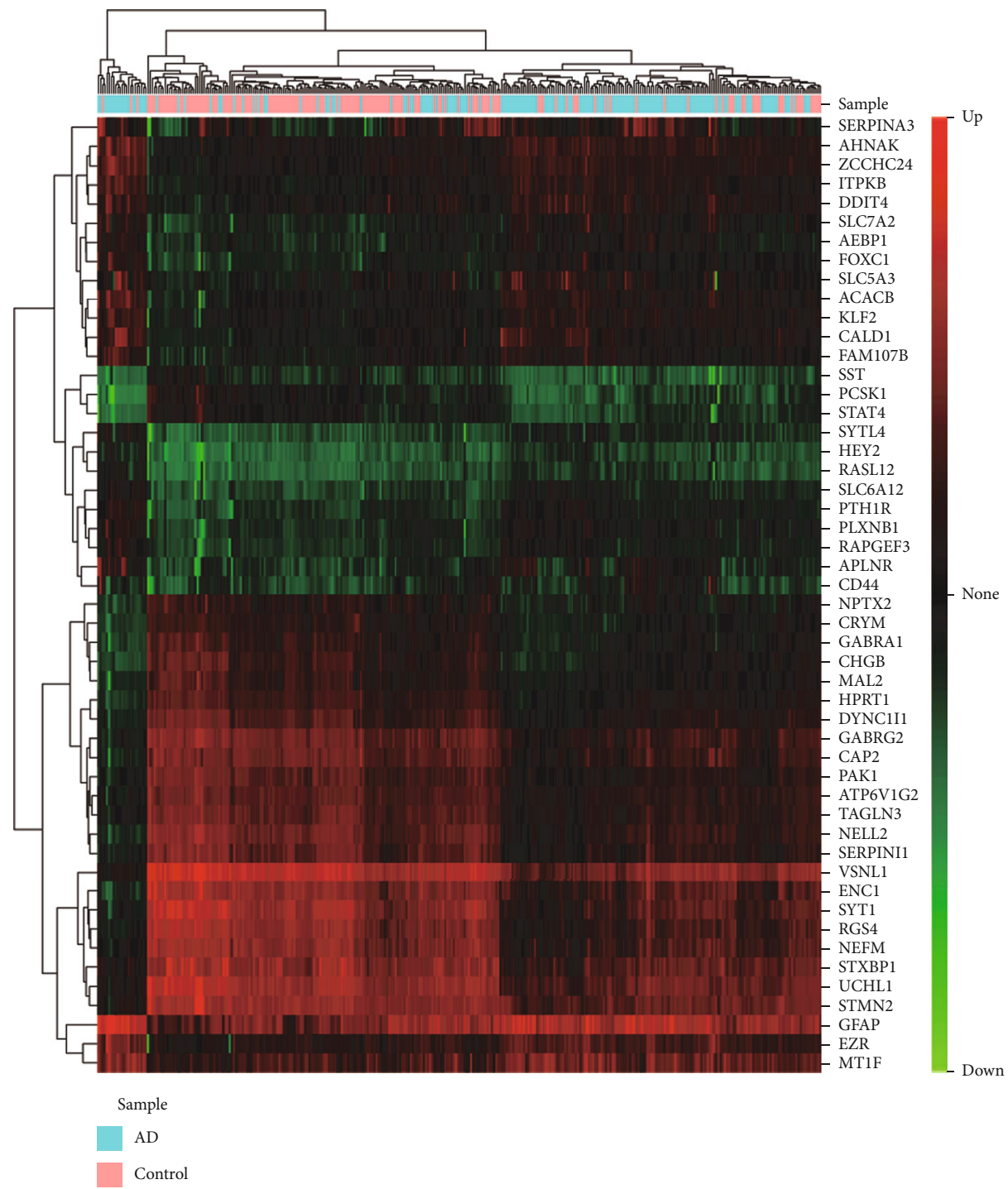

(b)

FIgURe 1: Differential expression analysis. (a) Volcano plot of DEGs between AD and nondementia controls. (b) Heatmap of the top 25 down- and upregulated DEGs: green to red indicates the process from down- to upregulation of gene expression. AD: Alzheimer's disease; DEGs: differentially expressed genes. 


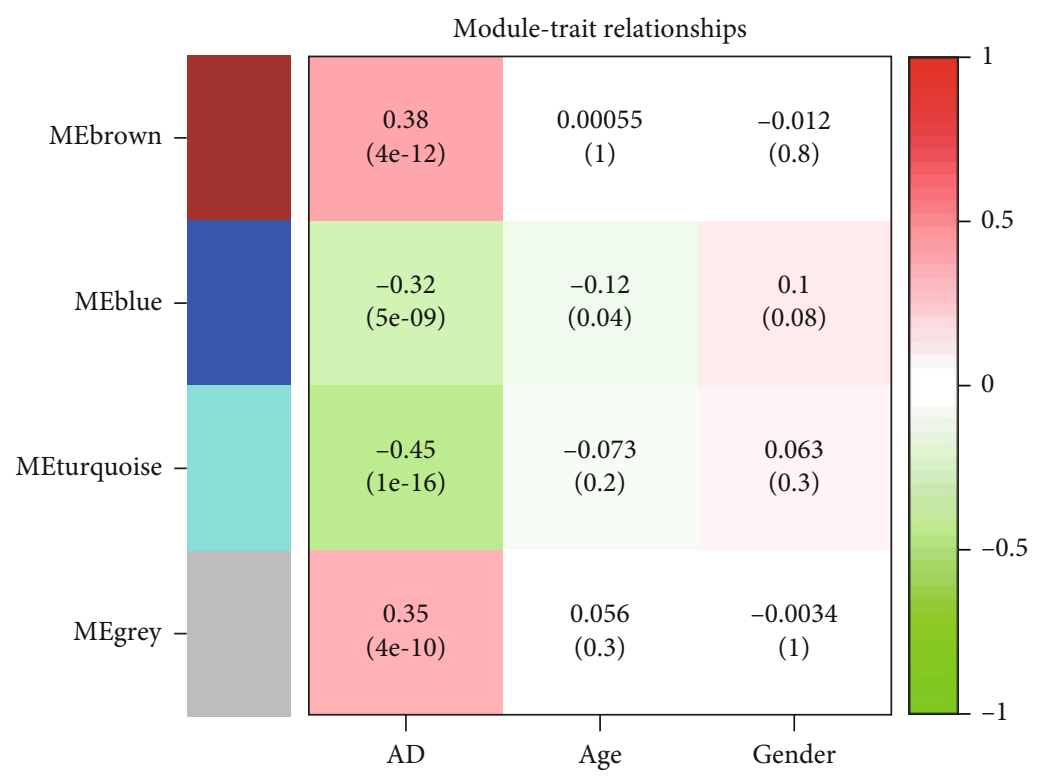

(a)

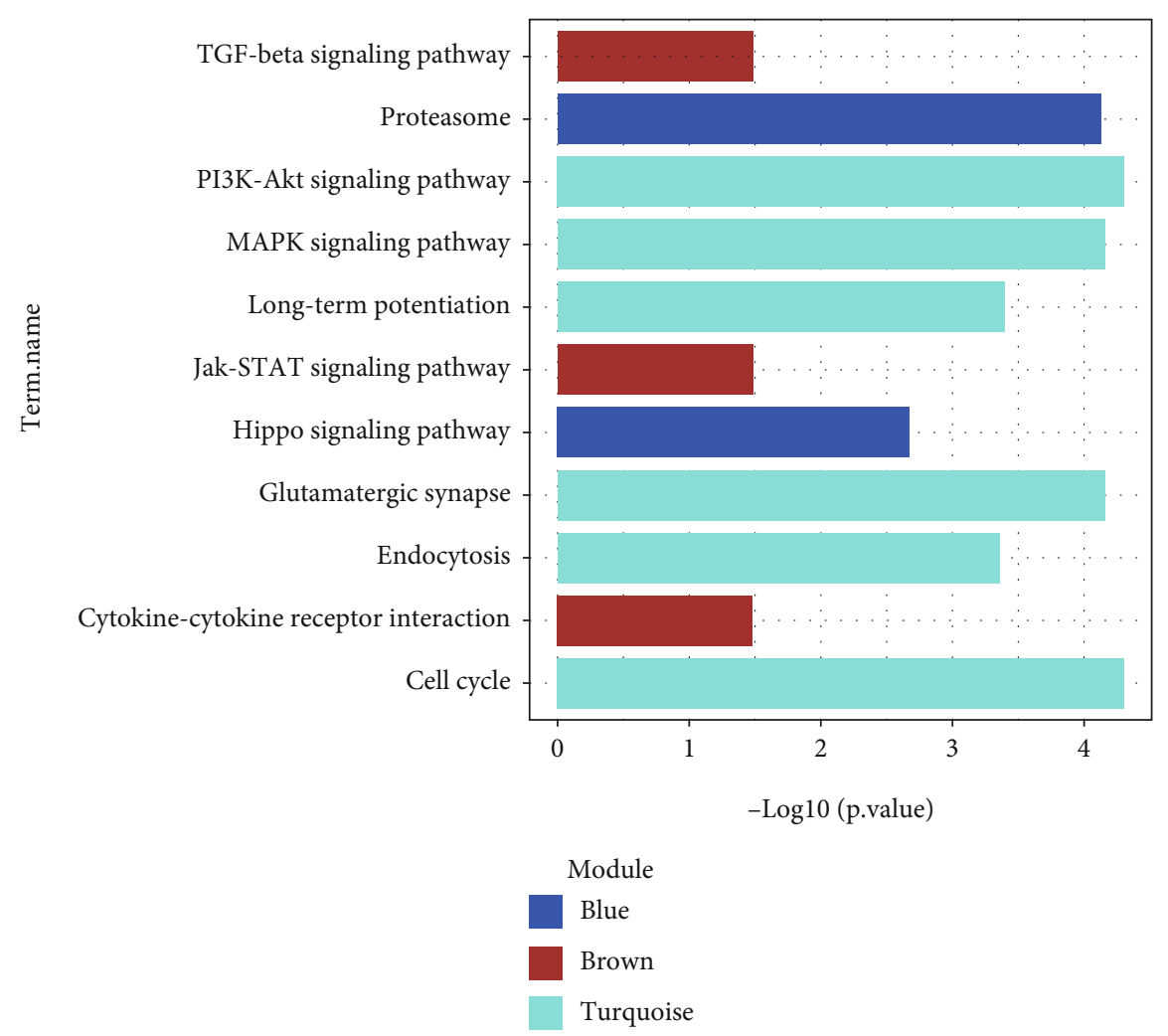

(b)

FIGURE 2: Weighted correlation network analysis. (a) Module-trait relationships of created modules: green to red represents the correlation of modules from negative to positive with phenotypes. (b) Enrichment of KEGG pathways on functional modules. AD: Alzheimer's disease; KEGG: Kyoto Encyclopedia of Genes and Genomes.

positive correlation of blue (correlation coefficient $=0.32$, $p=5 e-09$ ) and brown (correlation coefficient $=0.38, p=$ $4 e-12)$ modules with $\mathrm{AD}$ phenotype. Annotation of KEGG pathway (Figure 2(b)) was performed by functional enrichment analysis, which revealed that the DEGs in the blue module were involved in proteasome and Hippo sig- naling pathways; the DGEs of brown module participated in cytokine-cytokine receptor interaction, TGF-beta, and Jak-STAT signaling pathways; the DEGs in the turquoise module were enriched in cell cycle, endocytosis, glutamatergic synapse, long-term potentiation, PI3K-Akt, and MAPK signaling pathways. 


\begin{tabular}{|c|c|c|c|c|c|c|c|c|c|c|c|c|c|c|c|c|c|}
\hline (a) & - & (ल) & (BRCI) & (aiv) & (2) & त्रिलो & (Iin) & UBEAA & लिखे & $\infty$ & (reng) & $\infty$ & (10) & (nastr) & (2iㅏㅇㅛ & (iin) & rasers \\
\hline (a) & (orr) & (100) & (ar) & (avi) & (mot) & (max) & (-) & (nor) & (ex) & (cana) & (rarer) & $\infty$ & (a) & (2002) & RAD238 & (लriv) & लिखित्व \\
\hline (100) & (6⿴囗十) & 0 & (DRe) & Ferep & (5MB) & (rmas) & (2) & $\mathrm{Acms}$ & (MSH2) & (ioner & (iin) & 맥ㄱ & ResG6kg) & (1007) & (SIBP) & (100) & (SMA) \\
\hline (3i) & (Circ) & ( & 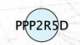 & (갠. & (Ba) & (20x6) & (niner & (OCl2) & Enze & (5003) & (i) & (ix) & (rect) & -iso & (2122) & (vir) & (ixir) \\
\hline mo & (ara) & (1313) & (Bxic) & (1015) & (ब) & (aia) & (ax) & (1) & (naie & ( & $\Leftrightarrow$ & (RPA3) & (exins? & (@) & (a) & (2A120) & (아앙 \\
\hline (rext) & (imes) & $\therefore$ & (실 & (1004) & (nass) & (FCB & (eme) & (nat) & (rine) & $\approx$ & (GF2) & $\Theta$ & (nerer & (RREA) & (स्थित्र & Pित्रे & (ear) \\
\hline ENPE: & HISTHZgo & (m) & (i) & $(x)$ & (아) & 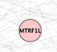 & (3ax) & (x) & (N1158) & (xara) & (a) & germa & UBE2N & 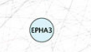 & (6xx) & (2000) & (MII) \\
\hline (3010) & (사라 & conkry & SSMB & (3ain) & $\infty$ & (5iII) & $\Leftrightarrow$ & NFFB & (a) & 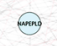 & 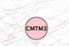 & (ai) & (a.) & HISTHसBEE & (सrase & $\Leftrightarrow$ & reate \\
\hline (200) & (an) & $\infty$ & (ii) & (a) & (ia) & (200) & (6orr) & 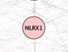 & (i.) & (BMI) & (3) & (min) & СКNㅛ & (3) & (a) & (ia) & MCM? \\
\hline (-) & (aii) & BTRC & (a) & (rim) & (2018) & (iii) & (coms & (rate & RABIA & PSMB? & (ळ) & (ima) & (Ix) & (xaz) & $(10)$ & (106) & (다알 \\
\hline (cati) & (aw) & $(30)$ & Norcar & (Ant21) & $\infty$ & (ini) & (11i1) & CDK2 & PSMBg & (RIA) & $(10)$ & (min) & (a) & (a) & (mam) & (x) & (5) \\
\hline (1060) & (탕 & $\therefore$ & (ixa) & (2007) & СØKN) & (nowar) & Perces & Hiserald & (PRCE) & (x) & (1002) & (10017) & (nex) & 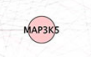 & (106) & (mix) & (ima) \\
\hline TNFPSFA & ADRB? & (DK4 & (2xat) & स्रिण & (x) & (20ix) & (m) & (NER2) & (ER & (sNeren) & (nis) & (CUIS) & (HRPF) & (ac) & $\Leftrightarrow$ & (2010) & 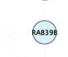 \\
\hline (as) & (1) & $\infty$ & (iai) & (maces) & tame & (nNx) & (DC) & Nupse & (iiii) & (2MPr) & (CIA) & (min) & 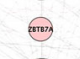 & (-) & $\Leftrightarrow$ & (3ang) & (mane) \\
\hline (아라) & SP1 & (erec) & 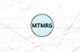 & (저소 & (min) & (2i) & $(3)$ & (iii) & $\mathrm{MCM}_{3}$ & (i) & (1)00 & $(\approx x)$ & BRCA 1 & (act) & (2a) & सिख्ध & (1) \\
\hline (स्2827) & (1017) & anarece & reange & (20) & (*) & (2) & (ख) & (x) & $(\infty x)$ & (ख) & 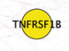 & (na) & (Irate) & EDogl & (200) & $(-\infty)$ & $\infty$ \\
\hline (now) & 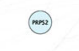 & (iㅏ맘 & (nax) & (wax) & MिCMG & (an) & (an) & - & $\infty$ & (PIR4) & $\Leftrightarrow$ & (ing & (1)ing & $\infty$ & - & (in) & (a) \\
\hline (a) & $\Leftrightarrow$ & (-) & (14ix) & $\infty$ & ल상 & $\infty$ & (nonc) & (iin) & (mir) & (im & (2068) & (iar) & - & (1000) & $-\infty$ & encer & (henz: \\
\hline (300) & - & (ex) & (int & 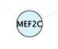 & $(i ;)$ & (a) & (1) & 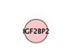 & $\infty$ & BARDD & & & & & & & \\
\hline
\end{tabular}

FIGURE 3: Global regulatory network. Global regulatory network of cell cycle-related genes: yellow represents the signature trophic receptors; blue indicates low expression; red represents high expression; node size reflects the degree of gene connectivity.

3.3. Module-Pathway Regulatory Network and AUC Analysis. Ten signature receptors (GRIN2A, GRIA2, CHRM1, GABRG2, PGRMC1, EPHA4, MAGED1, TNFRSF1B, TNFRSF1A, and RXRA) interacting with cell cycle genes were displayed in the global regulation network (Figure 3), thus to enrich the cross-talking pathways of signature receptors in AD. As shown in Figure 4, GRIN2A (i.e., glutamate ionotropic receptor NMDA type subunit $2 \mathrm{~A}$ ) belonging to a family of glutamate-gated ion channel receptors, participated in the glutamatergic synapse, long-term potential, cAMP, and calcium signaling pathways. GRIA2 (i.e., glutamate ionotropic receptor AMPA type subunit 2) encodes a member of the glutamate receptor family that is activated in numerous neuropathological processes [37-39]. This gene was involved in the dopaminergic synapse, long-term potential, and calcium signaling pathway. CHRM1 (i.e., cholinergic receptor muscarinic 1) belongs to a muscarinic cholinergic receptor in the larger family of $G$ protein-coupled receptors, which was enriched in PI3K-Akt, cAMP, and calcium signaling pathways. GABRG2 (i.e., gamma-aminobutyric acid type A receptor subunit gamma2) encodes a receptor of gammaaminobutyric acid (GABA) that is the major inhibitory neurotransmitter in the mammalian brain [40]. This gene participated in retrograde endocannabinoid signaling and nicotine addiction. RXRA (i.e., retinoid X receptor alpha) is a nuclear receptor mediating the biological effects of retinoids by participating in retinoic acid-mediated gene activation. The functional enrichment analysis showed this gene enriched in the PI3K-Akt signaling pathway. TNFRSF1A (i.e., TNF receptor superfamily member $1 \mathrm{~A}$ ), belonging to a member of the TNF receptor superfamily, was involved in MAPK, TNF, and NF-Kappa B signaling pathways. TNFRSF1B (i.e.,
TNF receptor superfamily member $1 \mathrm{~B}$ ) encoding another member of the TNF-receptor superfamily of proteins, participated in TNF signaling pathway. In brief, the signature receptors are mainly involved in the glutamatergic synapse (GRIN2A), long-term potential (GRIN2A, GRIA2), MAPK (TNFRSF1A), and PI3K-Akt (CHRM1, RXRA) signaling pathways (Figure 4). Analysis of AUC exhibited a good diagnostic performance of each signature receptor in predicting $\mathrm{AD}$ onset (Figure 5).

\section{Discussion}

To examine the relevance of the cell cycle in the AD brain, we took advantage of publicly available RNA-Seq data from 336 postmortem human samples to identify 775 cell cycle genes and 77 trophic receptors that were differentially expressed between $\mathrm{AD}$ and nondementia controls. The reason for choosing temporal lobe tissues for our investigation was on basis of the region's high susceptibility to neuron loss during neurodegeneration of $\mathrm{AD}$ [41]. Analytic results of GSEA showed that DEGs of the cell cycle were enriched in regulation of cell proliferation, programmed cell death, and apoptotic processes, as well as negative regulation of nucleic acid-templated transcription, RNA biosynthetic, and metabolic processes. Of note, these biological processes apparently pointed towards a confrontational interaction between cell demise and proliferation, supporting the underlying manifestation of cell cycle dysfunction in the human AD brain.

The results emerging from WGCNA revealed that brown, blue, and turquoise modules were intimately associated with $\mathrm{AD}$, the DEGs of which were enriched in proteasome, cytokine-cytokine receptor interaction, glutamatergic 


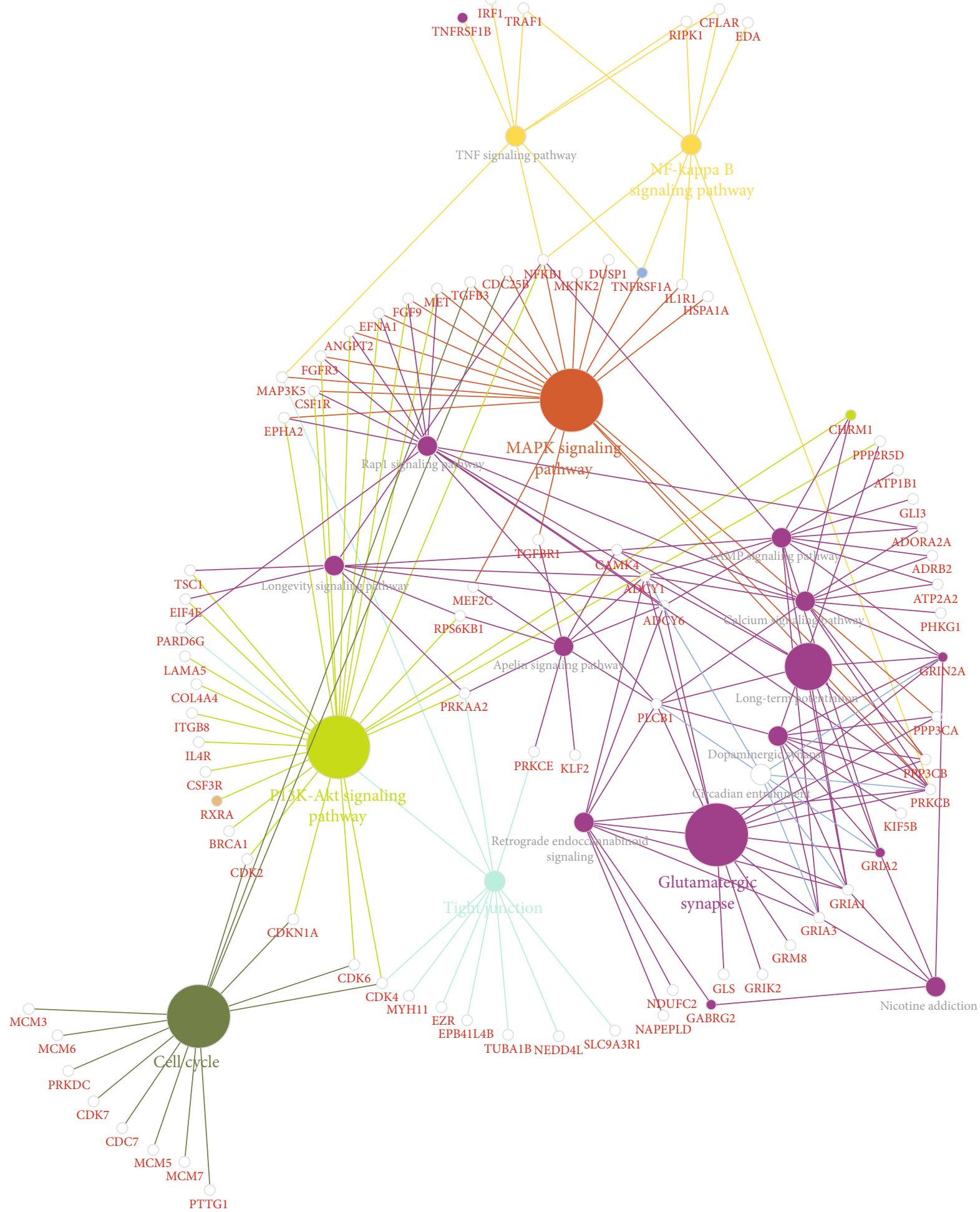

Figure 4: Cross-talking pathways. Cross-talking pathways of signature trophic receptors: small colored nodes indicate the signature trophic receptors.

synapse, long-term potentiation, endocytosis, PI3K-Akt, and MAPK signaling pathways. Accumulating evidence suggests that perturbations in the glutamatergic synapse, consisting of the presynaptic terminal, astrocytic process, and postsynaptic spine, underlie the pathogenic mechanisms of $\mathrm{AD}$
[42-44]. As the primary excitatory neurotransmitter, glutamate has been found to be implicated in hippocampusdependent learning and memory functions $[45,46]$. A $\beta$ induces extracellular glutamate release to provoke overexcitation of N-methyl-D-aspartate receptors (NMDARs), which 

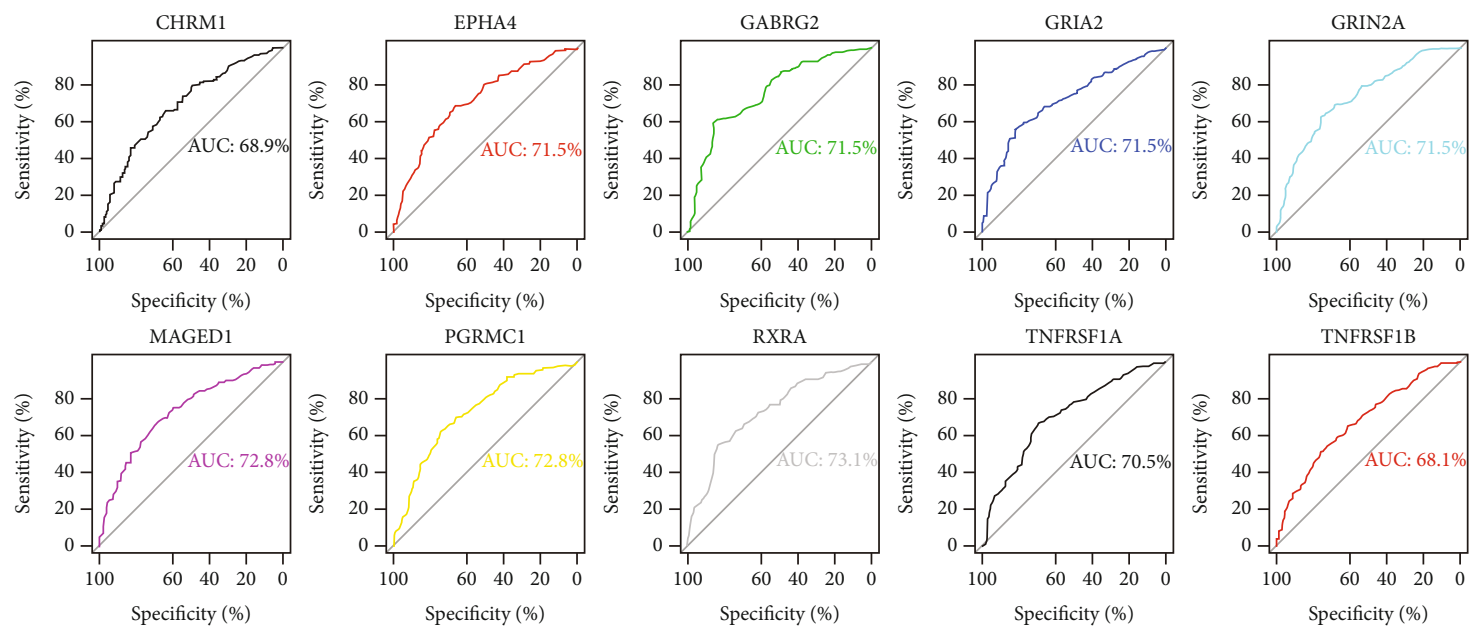

Figure 5: AUC analyses of trophic receptors. AUC: area under the curve.

is an excitotoxic process relevant to neuronal degeneration, cell damage, and apoptosis [47, 48]. Alternatively, this excitatory toxicity might also occur at the normal level of glutamate due to overstimulation of glutamate receptors, such as tau-induced alterations in NMDARs phosphorylation [49]. There were several pieces of evidence that glutamate bound to NMDARs to undergo a conformational change and thus to open ion channels [50-52]. The resultant influx of calcium initiated secondary messenger systems, giving rise to the induction of long-term potentiation, an activation process for synaptic plasticity and memory processing in hippocampus [53]. Evidence shows that either A $\beta$-induced glutamate release [54] or overactivation of NMDARs [33] has dramatic consequences for an overload of intracellular calcium, leading to impaired long-term potential, hyperphosphorylation of tau, and synaptic loss $[48,55]$.

In terms of MAPK and PI3K-Akt signaling, neurotrophin receptors couple with these pathways are responsible for many critical processes in $\mathrm{AD}$, as confirmed ranging from synaptic plasticity to neuronal growth and apoptosis [56]. The MAPK signaling constitutes a primary conduit to the genomic response of synaptically activated neurons essential for long-term recognition memory $[57,58]$. Specifically, oxidative stress induced by $\mathrm{A} \beta$ affects MAPK cascade activity, which inhibits astrocytic uptake of glutamate and upregulated NMDARs, resulting in cognitive impairment and $\mathrm{AD}$ neurodegeneration [59]. In addition, PI3K signaling was found to participate in the homeostatic regulation of apoptosis and memory consolidation by inhibiting proapoptotic transcription factors [60,61]. In the case of GSK3 $\beta$ factor, for instance, it is a ubiquitously expressed serine/threonine kinase interacting with extrasynaptic NMDARs in PI3K-Akt signaling, which contributes to tau phosphorylation, $\mathrm{A} \beta$ generation, and neuronal death, hence implying the involvement of trophic receptors in $\mathrm{AD}$ pathogenesis through the PI3K-Akt signaling pathway $[62,63]$.

We identified 10 signature trophic receptors (GRIN2A, GRIA2, CHRM1, GABRG2, PGRMC1, EPHA4, MAGED1,
TNFRSF1B, TNFRSF1A, and RXRA) suggestive of a robust association with $\mathrm{AD}$. The strong molecular and disease phenotypes from the created modules in our study determined these receptors as key regulators of $\mathrm{AD}$ pathogenesis, and that their mechanistic pathways were considered promising candidates for the treatment of AD. Some signature trophic receptors have already been approved or tested as drug targets for AD. GRIN2A encodes the GluN2A subunit of the NMDAR, which, according to its neuroexcitatory toxicity, is responsible for synaptic loss [64-66]. In view of this property, memantine, an NMDAR antagonist, has been developed for treating moderate-to-severe AD [67]. Deficient RNA editing of the GRIA2 Q/R site has been found to have pathogenic effects, which could be a target of novel therapeutic strategies in $\mathrm{AD}$ [68]. CHRM1 is a muscarinic cholinergic receptor that is considered another crucial antidementia target. In practice, acetylcholinesterase inhibitors (e.g., donepezil, rivastigmine, galantamine, and tacrine) have been widely used in the clinical treatment of mild-to-moderate $\mathrm{AD}[69$, 70]. Remarkably, all these signature trophic receptors exerted a good diagnostic performance in predicting $\mathrm{AD}$ according to AUC analysis, supporting them to be potential biomarkers of AD. Further in vivo or in vitro experiments are expected to validate the relevant pathways of the cell cycle underlying the pathological process of AD.

\section{Conclusions}

In aggregate, gene expression profiling is a promising approach to reveal the intricate mechanisms of the cell cycle underlying AD. Dysregulation of key trophic factor receptors in the cell cycle is involved in the pathogenesis of $\mathrm{AD}$, possibly mediated via glutamatergic synapse (GRIN2A), longterm potential (GRIN2A, GRIA2), MAPK (TNFRSF1A), and PI3K-Akt (CHRM1, RXRA) signaling pathways. Our findings illuminate the role of the cell cycle in AD neurodegeneration, which may fuel interest in the interaction of cell cycle regulation with $\mathrm{AD}$ therapy by targeting signature trophic receptors. 


\section{Data Availability}

The data (including GSE132903, GSE118553, GSE5281, GSE37264, and GSE36980) generated and/or analyzed in this study are openly available in GEO (https://www.ncbi.nlm.nih .gov/geo/).

\section{Conflicts of Interest}

The author(s) declare(s) that they have no conflicts of interest.

\section{Authors' Contributions}

CSZ, MZ, and ZKZ conceived and designed the study. JB, KXK, RWZ, SSZ, XQZ, and ZKZ conducted the experiments and analyzed the data. $\mathrm{MZ}$ and $\mathrm{ZKZ}$ wrote the original draft. CSZ, YX, and ZKZ reviewed and edited the paper. All authors reviewed and approved the final manuscript. Chuansheng Zhao and Mei Zhao contributed equally to this work.

\section{Acknowledgments}

The research is supported by the National Natural Science Foundation of China (No. 81372104), the Natural Science Foundation of Liaoning Province (No. 20180540150), the Shenyang Population and Health Technical Critical Special Project (No. F16-206-9-01), the Program of the Distinguished Professor of Liaoning Province (Chuansheng Zhao), and the Guidance plan for key research and development plans of Liaoning Province (No. 2019JH8/10300002).

\section{Supplementary Materials}

Supplementary 1. Supplementary Table S1: clinical phenotypic data of samples.

Supplementary 2. Supplementary Table S2: list of cell cycle genes.

Supplementary 3. Supplementary Table S3: list of trophic factor receptors.

Supplementary 4. Supplementary Table S4: differential expression of cell cycle-related genes.

Supplementary 5. Supplementary Table S5: PCC between cell cycle genes and trophic receptors. PCC: Pearson correlation coefficient.

\section{References}

[1] F. Leng and P. Edison, "Neuroinflammation and Microglial Activation in Alzheimer Disease: Where Do We Go from Here?," Nature Reviews Neurology, vol. 17, no. 3, pp. 157172, 2021.

[2] R. L. Neve and D. L. Mcphie, "The cell cycle as a therapeutic target for Alzheimer's disease," Pharmacology \& Therapeutics, vol. 111, no. 1, pp. 99-113, 2006.

[3] Z. Zhou, Y. Liang, X. Zhang et al., "Fibrinogen and risk of dementia: a systematic review and meta-analysis," Neuroscience and Biobehavioral Reviews, vol. 112, pp. 353-360, 2020.
[4] H. Braak and E. Braak, "Neuropathological stageing of Alzheimer-related changes," Acta Neuropathologica, vol. 82, no. 4, pp. 239-259, 1991.

[5] H. Malkki, "NGF gene therapy activates neurons in the AD patient brain," Nature Reviews. Neurology, vol. 11, no. 10, p. 548, 2015.

[6] S. J. B. Vos, F. Verhey, L. Frölich et al., "Prevalence and prognosis of Alzheimer's disease at the mild cognitive impairment stage,” Brain, vol. 138, no. 5, pp. 1327-1338, 2015.

[7] K. Herrup and Y. Yang, "Cell cycle regulation in the postmitotic neuron: oxymoron or new biology?," Nature Reviews Neuroscience, vol. 8, no. 5, pp. 368-378, 2007.

[8] E. Y. Lee, C. Y. Chang, N. Hu et al., "Mice deficient for Rb are nonviable and show defects in neurogenesis and haematopoiesis," Nature, vol. 359, no. 6393, pp. 288-294, 1992.

[9] C. Andorfer, C. M. Acker, Y. Kress, P. R. Hof, K. Duff, and P. Davies, "Cell-cycle reentry and cell death in transgenic mice expressing nonmutant human tau isoforms," The Journal of Neuroscience, vol. 25, no. 22, pp. 5446-5454, 2005.

[10] F. Huang, M. Wang, R. Liu et al., "Cdt2-controlled cell cycle reentry regulates the pathogenesis of Alzheimer's disease," Alzheimers Dement, vol. 15, no. 2, pp. 217-231, 2019.

[11] A. J. Obaya and J. M. Sedivy, "Regulation of Cyclin-Cdk activity in mammalian cells," Cellular and Molecular Life Sciences, vol. 59, no. 1, pp. 126-142, 2002.

[12] C. J. Sherr, "Mammalian $G_{1}$ cyclins," Cell, vol. 73, no. 6, pp. 1059-1065, 1993.

[13] R. Pentz, M. F. Iulita, A. Ducatenzeiler et al., "Nerve growth factor (Ngf) pathway biomarkers in down syndrome prior to and after the onset of clinical Alzheimer's disease: a paired Csf and plasma study," Alzheimer's \& Dementia, vol. 17, no. 4, pp. 605-617, 2021.

[14] Y. Lin, H. Liu, A. Waraky et al., "Sumo-modified insulin-like growth factor 1 receptor (Igf-1r) increases cell cycle progression and cell proliferation," Journal of Cellular Physiology, vol. 232, no. 10, pp. 2722-2730, 2017.

[15] R. Pentz, M. F. Iulita, A. Ducatenzeiler, D. A. Bennett, and A. C. Cuello, "The human brain Ngf metabolic pathway is impaired in the pre-clinical and clinical continuum of Alzheimers disease," Molecular Psychiatry, 2020.

[16] A. J. Westwood, A. Beiser, C. Decarli et al., "Insulin-like growth Factor-1 and risk of Alzheimer dementia and brain atrophy," Neurology, vol. 82, no. 18, pp. 16131619, 2014.

[17] T. Hochstrasser, D. Ehrlich, J. Marksteiner, B. Sperner-Unterweger, and C. Humpel, "Matrix metalloproteinase-2 and epidermal growth factor are decreased in platelets of Alzheimer patients," Current Alzheimer Research, vol. 9, no. 8, pp. 982 989, 2012.

[18] E. R. Mahoney, L. Dumitrescu, A. M. Moore et al., "Brain expression of the vascular endothelial growth factor gene family in cognitive aging and alzheimer's disease," Molecular Psychiatry, vol. 26, no. 3, pp. 888-896, 2021.

[19] S. Chen, S.-T. Chen, Y. Sun et al., "Fibroblast growth factor 21 ameliorates neurodegeneration in rat and cellular models of Alzheimer's disease," Redox Biology, vol. 22, article 101133, 2019.

[20] J. L. Urdiales, E. Becker, M. Andrieu et al., "Cell cycle phasespecific surface expression of nerve growth factor receptors Trka and p75NTR," The Journal of Neuroscience, vol. 18, no. 17, pp. 6767-6775, 1998. 
[21] T. Barrett, S. E. Wilhite, P. Ledoux et al., "Ncbi geo: archive for functional genomics data sets-update," Nucleic Acids Research, vol. 41, pp. D991-D995, 2013.

[22] G. Mckhann, D. Drachman, M. Folstein, R. Katzman, D. Price, and E. M. Stadlan, "Clinical diagnosis of Alzheimer's disease: report of the Nincds-Adrda work group under the auspices of Department of Health and Human Services Task Force on Alzheimer's disease," Neurology, vol. 34, no. 7, pp. 939-944, 1984.

[23] American Psychiatric Association, Diagnostic and Statistical Manual of Mental Disorders, American Psychiatric Association, Washington DC, 3rd edition, 1980.

[24] American Psychiatric Association, Diagnostic and Statistical Manual of Mental Disorders, American Psychiatric Association, Washington DC, 3rd edition, 1987.

[25] American Psychiatric Association, Diagnostic and Statistical Manual of Mental Disorders: Dsm-Iv, American Psychiatric Association, Washington Dc, 4th edition, 1994.

[26] American Psychiatric Association, Diagnostic and Statistical Manual of Mental Disorders, Dsm-5, American Psychiatric Association, Washington Dc, 5th edition, 2013.

[27] S. S. Mirra, A. Heyman, D. McKeel et al., "The consortium to establish a registry for Alzheimer's disease (Cerad). Part Ii. Standardization of the Neuropathologic Assessment of Alzheimer's Disease," Neurology, vol. 41, no. 4, pp. 479-486, 1991.

[28] G. D. Grant, L. Brooks, X. Zhang et al., "Identification of cell cycle-regulated genes periodically expressed in U2os cells and their regulation by Foxm1 and E2f transcription factors," Molecular Biology of the Cell, vol. 24, no. 23, pp. 3634-3650, 2013.

[29] V. K. Mootha, C. M. Lindgren, K.-F. Eriksson et al., "PGC-1 $\alpha$ responsive genes involved in oxidative phosphorylation are coordinately downregulated in human diabetes," Nature Genetics, vol. 34, no. 3, pp. 267-273, 2003.

[30] A. Subramanian, P. Tamayo, V. K. Mootha et al., "Gene set enrichment analysis: a knowledge-based approach for interpreting genome-wide expression profiles," Proceedings of the National Academy of Sciences of the United States of America, vol. 102, no. 43, pp. 15545-15550, 2005.

[31] C. W. Law, Y. Chen, W. Shi, and G. K. Smyth, "Voom: precision weights unlock linear model analysis tools for RnaSeq read counts," Genome Biology, vol. 15, no. 2, p. R29, 2014.

[32] M. E. Ritchie, B. Phipson, D. Wu et al., "Limma powers differential expression analyses for Rna-sequencing and microarray studies," Nucleic Acids Research, vol. 43, no. 7, article e47, 2015.

[33] F. N. Soria, A. Pérez-Samartín, A. Martin et al., "Extrasynaptic glutamate release through cystine/glutamate Antiporter contributes to ischemic damage," The Journal of Clinical Investigation, vol. 124, no. 8, pp. 3645-3655, 2014.

[34] P. Langfelder and S. Horvath, "Wgcna: an R package for weighted correlation network analysis," BMC Bioinformatics, vol. 9, no. 1, p. 559, 2008.

[35] D. Szklarczyk, J. H. Morris, H. Cook et al., “The string database in 2017: quality-controlled protein-protein association networks, made broadly accessible," Nucleic Acids Research, vol. 45, no. D1, pp. D362-D368, 2017.

[36] D. Otasek, J. H. Morris, J. Bouças, A. R. Pico, and B. Demchak, "Cytoscape automation: empowering workflow-based network analysis,” Genome Biology, vol. 20, no. 1, p. 185, 2019.
[37] C. F. Gasparini, H. G. Sutherland, L. M. Haupt, and L. R. Griffiths, "Genetic analysis of Gria2 and Gria4 genes in migraine," Headache, vol. 54, no. 2, pp. 303-312, 2014.

[38] X. Li, W. Chen, Q. Yu et al., "A circuit of mossy cells controls the efficacy of memory retrieval by Gria2i inhibition of Gria2," Cell Reports, vol. 34, no. 7, article 108741, 2021.

[39] V. Salpietro, C. L. Dixon, H. Guo et al., "Ampa receptor Glua2 subunit defects are a cause of neurodevelopmental disorders," Nature Communications, vol. 10, no. 1, article 3094, 2019.

[40] C.-Q. Zhang, B. Mcmahon, H. Dong et al., "Molecular basis for and chemogenetic modulation of comorbidities in Gabrg2deficient epilepsies," Epilepsia, vol. 60, no. 6, pp. 1137-1149, 2019.

[41] M. Grundman, J. Corey-Bloom, T. Jernigan, S. Archibald, and L. J. Thal, "Low body weight in Alzheimer's disease is associated with mesial temporal cortex atrophy," Neurology, vol. 46, no. 6, pp. 1585-1591, 1996.

[42] K. J. Barnham and A. I. Bush, "Metals in Alzheimer's and Parkinson's diseases," Current Opinion in Chemical Biology, vol. 12, no. 2, pp. 222-228, 2008.

[43] C. C. Rudy, H. C. Hunsberger, D. S. Weitzner, and M. N. Reed, "The role of the tripartite glutamatergic synapse in the pathophysiology of Alzheimer's disease," Aging and Disease, vol. 6, no. 2, pp. 131-148, 2015.

[44] M. S. Unger, E. Li, L. Scharnagl et al., " $\mathrm{CD} 8^{+}$T-cells infiltrate Alzheimer's disease brains and regulate neuronaland synapse-related gene expression in APP-PS1 transgenic mice," Brain, Behavior, and Immunity, vol. 89, pp. 67-86, 2020.

[45] D. R. Curtis, J. W. Phillis, and J. C. Watkins, "The chemical excitation of spinal neurones by certain acidic amino acids," The Journal of Physiology, vol. 150, no. 3, pp. 656-682, 1960.

[46] A. L. Sheldon and M. B. Robinson, "The role of glutamate transporters in neurodegenerative diseases and potential opportunities for intervention," Neurochemistry International, vol. 51, no. 6-7, pp. 333-355, 2007.

[47] T. P. Obrenovitch and J. Urenjak, "Altered glutamatergic transmission in neurological disorders: from high extracellular glutamate to excessive synaptic efficacy," Progress in Neurobiology, vol. 51, no. 1, pp. 39-87, 1997.

[48] M. Talantova, S. Sanz-Blasco, X. Zhang et al., “A $\beta$ induces astrocytic glutamate release, extrasynaptic Nmda receptor activation, and synaptic loss," Proceedings of the National Academy of Sciences of the United States of America, vol. 110, no. 27, pp. E2518-E2527, 2013.

[49] L. M. Ittner, Y. D. Ke, F. Delerue et al., "Dendritic function of tau mediates amyloid- $\beta$ toxicity in Alzheimer's disease mouse models," Cell, vol. 142, no. 3, pp. 387-397, 2010.

[50] C. Henneberger, T. Papouin, S. H. R. Oliet, and D. A. Rusakov, "Long-term potentiation depends on release of $\mathrm{D}$ serine from astrocytes," Nature, vol. 463, no. 7278, pp. 232-236, 2010.

[51] C. E. Jahr and C. F. Stevens, "Calcium permeability of the Nmethyl-D-aspartate receptor channel in hippocampal neurons in culture," Proceedings of the National Academy of Sciences of the United States of America, vol. 90, no. 24, pp. 11573-11577, 1993.

[52] A. Panatier, D. T. Theodosis, J.-P. Mothet et al., "Glia-derived D-serine controls Nmda receptor activity and synaptic memory," Cell, vol. 125, no. 4, pp. 775-784, 2006. 
[53] T. V. Bliss and G. L. Collingridge, "A synaptic model of memory: long-term potentiation in the hippocampus," Nature, vol. 361, no. 6407, pp. 31-39, 1993.

[54] J. Brito-Moreira, A. C. Paula-Lima, T. R. Bomfim et al., “A $\beta$ oligomers induce glutamate release from hippocampal neurons," Current Alzheimer Research, vol. 8, no. 5, pp. 552-562, 2011.

[55] C. Tackenberg, S. Grinschgl, A. Trutzel et al., "Nmda receptor subunit composition determines beta-amyloid-induced neurodegeneration and synaptic loss," Cell Death \& Disease, vol. 4, no. 4, article e608, 2013.

[56] F. Guillot, S. Kemppainen, G. Lavasseur et al., "Brain-specific basal and novelty-induced alternations in Pi3k-Akt and Map$\mathrm{k} /$ Erk signaling in a middle-aged $\mathrm{A} \beta \mathrm{pp} / \mathrm{Ps} 1$ mouse model of Alzheimer's disease," Journal of Alzheimer's Disease, vol. 51, no. 4, pp. 1157-1173, 2016.

[57] B. Bozon, A. Kelly, S. A. Josselyn, A. J. Silva, S. Davis, and S. Laroche, "Mapk, Creb and Zif268 are all required for the consolidation of recognition memory," Philosophical Transactions of the Royal Society of London. Series B, Biological Sciences, vol. 358, no. 1432, pp. 805-814, 2003.

[58] S. Davis and S. Laroche, "Mitogen-activated protein kinase/extracellular regulated kinase signalling and memory stabilization: a review," Genes, Brain, and Behavior, vol. 5, pp. 61-72, 2006.

[59] M. Matos, E. Augusto, C. R. Oliveira, and P. Agostinho, "Amyloid-beta peptide decreases glutamate uptake in cultured astrocytes: involvement of oxidative stress and mitogen-activated protein kinase cascades," Neuroscience, vol. 156, no. 4, pp. 898-910, 2008.

[60] J. M. Horwood, F. Dufour, S. Laroche, and S. Davis, "Signalling mechanisms mediated by the phosphoinositide 3-kinase/Akt cascade in synaptic plasticity and memory in the rat," The European Journal of Neuroscience, vol. 23, no. 12, pp. 33753384, 2006.

[61] X. Zhang, N. Tang, T. J. Hadden, and A. K. Rishi, "Akt, Foxo and regulation of apoptosis," Biochimica et Biophysica Acta, vol. 1813, no. 11, pp. 1978-1986, 2011.

[62] Z. Cai, Y. Zhao, and B. Zhao, "Roles of glycogen synthase kinase 3 in Alzheimer's disease," Current Alzheimer Research, vol. 9, no. 7, pp. 864-879, 2012.

[63] F. Hernandez, J. J. Lucas, and J. Avila, "Gsk3 and tau: two convergence points in Alzheimer's disease," Journal of Alzheimer's Disease, vol. 33, no. s1, pp. S141-S144, 2012.

[64] G. E. Hardingham, "Targeting synaptic Nmda receptor coagonism as a therapy for Alzheimer's disease?," Cell Metabolism, vol. 31, no. 3, pp. 439-440, 2020.

[65] K. Marwick, P. Skehel, G. Hardingham, and D. Wyllie, "Effect of a Grin2a De novo mutation associated with epilepsy and intellectual disability on Nmda receptor currents and mg2+ block in cultured primary cortical neurons," The Lancet, vol. 385, p. S65, 2015.

[66] W.-Y. Ong, K. Tanaka, G. S. Dawe, L. M. Ittner, and A. A. Farooqui, "Slow excitotoxicity in Alzheimer's disease," Journal of Alzheimer's Disease, vol. 35, no. 4, pp. 643-668, 2013.

[67] S. A. Lipton, "Paradigm shift in neuroprotection by Nmda receptor blockade: memantine and beyond," Nature Reviews. Drug Discovery, vol. 5, no. 2, pp. 160-170, 2006.
[68] I. Gaisler-Salomon, E. Kravitz, Y. Feiler et al., "Hippocampusspecific deficiency in Rna editing of Glua2 in Alzheimer's disease," Neurobiology of Aging, vol. 35, no. 8, pp. 1785-1791, 2014.

[69] A. Fisher, "Cholinergic treatments with emphasis on M1 muscarinic agonists as potential disease-modifying agents for Alzheimer's disease," Neurotherapeutics, vol. 5, no. 3, pp. 433$442,2008$.

[70] P. M. Kemp, C. Holmes, S. Hoffmann et al., “A randomised placebo controlled study to assess the effects of cholinergic treatment on muscarinic receptors in Alzheimer's disease," Journal of Neurology, Neurosurgery, and Psychiatry, vol. 74, no. 11, pp. 1567-1570, 2003. 